\title{
Sinusoidal Response of a Second-order Digital Filter with Two's Complement Arithmetic
}

\author{
Bingo Wing-Kuen Ling and Peter Kwong-Shun Tam, Member, IEEE
}

\begin{abstract}
In this paper, results of the sinusoidal response case are presented. It is found that the visual appearance of the trajectory of the sinusoidal response case is much richer than that of the autonomous and step response cases. Based on the state space technique, the state vectors to be periodic are investigated. The set of initial conditions and the necessary conditions on the filter parameters are also derived. When overflow occurs, the system is nonlinear. If the corresponding symbolic sequences are periodic, some trajectory patterns are simulated. Since the state space technique is not sufficient to efficiently derive the sets of initial conditions and the necessary conditions on the filter parameters, a frequency-domain technique is employed to figure out the set of initial conditions. When the symbolic sequences are aperiodic, an elliptical fractal pattern or random-like chaotic pattern is found.
\end{abstract}

Index Terms-Sinusoidal response, chaotic behavior, second-order digital filter with two's complement arithmetic

\section{INTRODUCTION}

Many researchers had studied the chaotic behavior of an autonomous system [1]. 
Some trajectory equations and sets of initial conditions corresponding to some types of trajectories are characterized in [1]. However, practically, various types of input signals are usually applied. As a result, we need to analyze forced-input systems in addition to autonomous systems. For the step-input case, some new analytical and simulation results analogous to the autonomous case can be obtained by the method of affine transformation [2]. In this paper, we concentrate on the analysis of systems with sinusoidal inputs. In this case, the method of affine transformation is not sufficient to efficiently explain the trajectory behaviors, and other techniques, such as the frequency-domain technique, have to be employed as well.

\section{SYSTEM DESCRIPTION}

Following [1], [2], the state space model of a direct form second-order digital filter with two's complement arithmetic can be modeled as

$$
\mathbf{x}(k+1)=\left[\begin{array}{c}
x_{2}(k) \\
f\left(b \cdot x_{1}(k)+a \cdot x_{2}(k)+u(k)\right)
\end{array}\right]=\mathbf{A} \cdot \mathbf{x}(k)+\mathbf{B} \cdot u(k)+\left[\begin{array}{l}
0 \\
2
\end{array}\right] \cdot s(k) .
$$

In this paper, we only consider the case when the filter is marginally stable, that is $b=-1$ and $|a|<2$. The external input is represented as $u(k)=c \cdot \sin (\theta \cdot k) \cdot v(k)$ for $c \in \mathfrak{R} \backslash\{0\}, \quad \theta \in \mathfrak{R} \backslash\{k \cdot \pi: k \in Z\}$, and $v(k)$ is the unit step function. When $c=0$ or $\theta=k \cdot \pi$, where $k \in \mathbf{Z}$, the input signal is zero everywhere and the system reduces to the autonomous response case, for which detail analysis can be found in [1].

The behaviors of the state trajectories of the autonomous response are reported in [1]. Based on the method of affine transformation, new results can also be derived for the step response case [2]. However, is the behavior for the sinusoidal response 
similar to those of the autonomous and step response cases? What are the trajectory patterns on the phase portrait? What are the sets of initial conditions for the different types of trajectories? How do the filter and input parameters affect the trajectory behavior?

\section{ANALYTICAL AND SIMULATION RESULTS FOR NON-OVERFLOW}

\section{CASE}

\section{A. Trajectory equation}

Define $\quad \cos \Omega=\frac{a}{2} \quad, \quad \mathbf{T}_{\Omega}=\left[\begin{array}{cc}1 & 0 \\ \cos \Omega & \sin \Omega\end{array}\right], \quad \mathbf{T}_{\theta}=\left[\begin{array}{cc}1 & 0 \\ \cos \theta & \sin \theta\end{array}\right]$, $\widehat{\mathbf{A}}_{\Omega}=\left[\begin{array}{cc}\cos \Omega & \sin \Omega \\ -\sin \Omega & \cos \Omega\end{array}\right], \quad \widehat{\mathbf{A}}_{\theta}=\left[\begin{array}{cc}\cos \theta & \sin \theta \\ -\sin \theta & \cos \theta\end{array}\right]$ and $\mathbf{x}_{1}{ }^{*}=\frac{c \cdot \sin \theta}{2 \cdot \cos \theta-a} \cdot\left[\begin{array}{l}1 \\ 0\end{array}\right]$. Since $|a|<2, \Omega$ is not integer multiples of $\pi$, so $\mathbf{T}_{\Omega}^{-1}$ exists. As $\theta \neq k \cdot \pi$, so $\mathbf{T}_{\theta}^{-1}$ also exists. Hence, we have $\mathbf{x}(k)=\mathbf{T}_{\Omega} \cdot \widehat{\mathbf{A}}_{\Omega}{ }^{k} \cdot \mathbf{T}_{\Omega}{ }^{-1} \cdot\left(\mathbf{x}(0)+\mathbf{x}_{1}{ }^{k}\right)-\mathbf{T}_{\theta} \cdot \widehat{\mathbf{A}}_{\theta}{ }^{k} \cdot \mathbf{T}_{\theta}{ }^{-1} \cdot \mathbf{x}_{1}{ }^{*}$ for $k \geq 1$.

Since $\mathbf{x}(k)$ is a superposition of two signals with different frequencies, this causes a rich set of trajectory patterns on the phase portrait. Figure 1 shows some examples of the different types of trajectories. As shown in Figure 1, the trajectory patterns are different from, and more complicated than those of the autonomous and step response cases.

It can be also seen from Figure 1.1 that there are several ellipses on the phase portrait even when no overflow occurs. This trajectory pattern seems to correspond to the type II trajectory (overflow case) for the autonomous and step response cases [1], [2]. However, overflow does not occur for the sinusoidal response case. Hence, we cannot classify the types of trajectories based on the visual appearances of the 
trajectories. As a result, we have to employ other properties, such as the periodicity of symbolic sequences, for the classification of the types of trajectories.

\section{B. Periodicity of state vector}

Because $\mathbf{x}(k)$ is made up of two different components with frequencies $\Omega$ and $\theta$, the frequency spectrum of $\mathbf{x}(k)$ consists of a finite set of impulses. If $\Omega$ and $\theta$ are rational multiples of $\pi$, that is, $\Omega=p_{1} \cdot \pi$ and $\theta=p_{2} \cdot \pi$, for $p_{1}, p_{2} \in \mathrm{Q}$, then $\mathbf{x}(k)$ is periodic. So, there are finitely many distinct points on the trajectory as shown in Figure 2.1. However, if $\Omega$ or $\theta$ are not rational multiples of $\pi$, then $\mathbf{x}(k)$ is aperiodic. Hence, there are infinitely many distinct points on the trajectory as shown in Figure 2.2.

Compared to the autonomous and step response cases, $\mathbf{x}(k)$ is made up of a component with frequency $\Omega$ and a DC component. If $\Omega$ is a rational multiple of $\pi$, that is, $\Omega=p_{1} \cdot \pi$, for $p_{1} \in \mathrm{Q}$, then $\mathbf{x}(k)$ is periodic. So, there are finitely many distinct points on the ellipse. However, if $\Omega$ is not a rational multiple of $\pi$, then there are infinitely many distinct points on the ellipse [1]-[3].

\section{Set of initial conditions}

It was found that the set of initial conditions for the autonomous and step response cases when no overflow occurs can be described by an elliptical region [1], [2]. We found a similar result for the sinusoidal response. As no overflow occurs, we have

$$
\| \mathbf{T}_{\Omega}^{-1} \cdot\left(\mathbf{x}(0)+\mathbf{x}_{1}^{*}\|\leq 1-\| \mathbf{T}_{\theta}^{-1} \cdot \mathbf{x}_{1}^{*} \| .\right.
$$

Hence, the set of initial conditions for the non-overflow case is

$$
\left\{\mathbf{x}(0):\left\|\mathbf{T}_{\Omega}^{-1} \cdot\left(\mathbf{x}(0)+\mathbf{x}_{1}^{*}\right)\right\| \leq 1-\left\|\mathbf{T}_{\theta}^{-1} \cdot \mathbf{x}_{1}^{*}\right\|\right\} .
$$


This set of initial conditions can be represented as an elliptical region in the phase portrait diagram. The center of the ellipse is located at $-\mathbf{x}_{1}{ }^{*}$ and the size of the ellipse depends on $1-\left\|\mathbf{T}_{\theta}^{-1} \cdot \mathbf{x}_{1}^{*}\right\|$. Figure 3 shows an example of the set of initial conditions for the situation when overflow does not occur.

\section{Necessary conditions on the filter parameters}

For the autonomous response case, there always exist some initial conditions for any filter parameter in the set $a \in\{a:|a|<2\}$ such that the system is free from overflow [2]. However, this is not true for the sinusoidal response case because there are two more parameters from the input signal, $c$ and $\theta$. In fact, we have found that if the set of filter and input parameters fails to satisfy a particular relation, then overflow has to occur no matter what the initial conditions are.

From the inequality (3), we have

$$
\left\|\mathbf{T}_{\Omega}{ }^{-1} \cdot\left(\mathbf{x}(0)+\mathbf{x}_{1}{ }^{*}\right)\left|\leq 1-\left\|\mathbf{T}_{\theta}^{-1} \cdot \mathbf{x}_{1}^{*}\right\|=1-\right| \frac{c}{2 \cdot(\cos \Omega-\cos \theta)} \mid .\right.
$$

Hence, we have

$$
\frac{|c|}{2} \leq|\cos \Omega-\cos \theta| \text {. }
$$

If the relation (6) is not satisfied, overflow has to occur no matter what the initial conditions are. Figure 4 shows the region of the parameter space that satisfies the relation (6).

From the analysis, if $\theta=2 \cdot \pi \cdot m \pm \Omega$, then $c$ has to be zero. Therefore, overflow will occur no matter what the initial conditions are, even when a very small signal $(c \rightarrow 0$, where $|c|>0)$ is applied to the system. This phenomenon can be understood by the resonance behavior. 


\section{ANALYTICAL AND SIMULATION RESULTS FOR OVERFLOW CASE}

\section{A. Trajectory equation}

For the autonomous and step response cases, there are more than one ellipses on the phase portrait [1], [2] if the symbolic sequence is periodic. However, the sinusoidal response case is more complicated than that of the autonomous and step response cases, as discussed below.

Assume the periodic sequences are periodic with period $M$. Let

$$
\begin{aligned}
& \mathbf{x}_{1, p}{ }^{*}=\frac{a_{p} \cdot \sin (p \cdot \omega)}{\cos (p \cdot \omega)-\cos \Omega} \cdot\left[\begin{array}{l}
1 \\
0
\end{array}\right], \quad, \quad \mathbf{x}_{2, p}{ }^{*}=\frac{b_{p}}{\cos (p \cdot \omega)-\cos \Omega} \cdot\left[\begin{array}{cc}
\cos (p \cdot \omega) \\
1
\end{array}\right], \\
& \mathbf{T}_{p \cdot \omega}=\left[\begin{array}{cc}
1 & 0 \\
\cos (p \cdot \omega) & \sin (p \cdot \omega)
\end{array}\right], \quad \widehat{\mathbf{A}}_{p \cdot \omega}=\left[\begin{array}{cc}
\cos (p \cdot \omega) & \sin (p \cdot \omega) \\
-\sin (p \cdot \omega) & \cos (p \cdot \omega)
\end{array}\right], \quad \text { for } \\
& p=0,1, \cdots, M-1, \text { and } \mathbf{x}_{2}{ }^{*}=\frac{b_{0}}{1-\cos \Omega} \cdot\left[\begin{array}{l}
1 \\
1
\end{array}\right] .
\end{aligned}
$$

If $M$ is odd, then we have

$$
\begin{aligned}
& \mathbf{x}(k)=\mathbf{T}_{\Omega} \cdot \widehat{\mathbf{A}}_{\Omega}{ }^{k} \cdot \mathbf{T}_{\Omega}{ }^{-1} \cdot\left(\mathbf{x}(0)+\mathbf{x}_{1}{ }^{*}-\mathbf{x}_{2}{ }^{*}+\sum_{p=1}^{M-1}\left(\mathbf{x}_{1, p}{ }^{*}-\mathbf{x}_{2, p}{ }^{*}\right)\right)-\mathbf{T}_{\theta} \cdot \widehat{\mathbf{A}}_{\theta}{ }^{k} \cdot \mathbf{T}_{\theta}{ }^{-1} \cdot \mathbf{x}_{1}{ }^{*}+ \\
& \sum_{p=1}^{M-1} \mathbf{T}_{p \cdot \omega} \cdot \widehat{\mathbf{A}}_{p \cdot \omega}{ }^{k} \cdot \mathbf{T}_{p \cdot \omega}{ }^{-1} \cdot\left(\mathbf{x}_{2, p}{ }^{*}-\mathbf{x}_{1, p}{ }^{*}\right)+\mathbf{x}_{2}{ }^{*} \\
& \text { for } k \geq 1 .
\end{aligned}
$$

If $M$ is even, let $\mathbf{x}_{3}{ }^{*}=\frac{b_{\frac{M}{2}}}{1+\cos \Omega} \cdot\left[\begin{array}{c}-1 \\ 1\end{array}\right]$, then we have

$$
\begin{aligned}
& \mathbf{x}(k)=\mathbf{T}_{\Omega} \cdot \widehat{\mathbf{A}}_{\Omega}{ }^{k} \cdot \mathbf{T}_{\Omega}{ }^{-1} \cdot\left(\mathbf{x}(0)+\mathbf{x}_{1}{ }^{*}-\mathbf{x}_{2}{ }^{*}+\mathbf{x}_{3}{ }^{*}+\sum_{\substack{p=1 \\
p \neq \frac{M}{2}}}^{M-1}\left(\mathbf{x}_{1, p}{ }^{*}-\mathbf{x}_{2, p}{ }^{*}\right)\right)-\mathbf{T}_{\theta} \cdot \widehat{\mathbf{A}}_{\theta}{ }^{k} \cdot \mathbf{T}_{\theta}{ }^{-1} \cdot \mathbf{x}_{1}{ }^{*}+ \\
& \sum_{\substack{p=1 \\
p \neq \frac{M}{2}}}^{M-1} \mathbf{T}_{p \cdot \omega} \cdot \widehat{\mathbf{A}}_{p \cdot \omega}{ }^{k} \cdot \mathbf{T}_{p \cdot \omega}{ }^{-1} \cdot\left(\mathbf{x}_{2, p}{ }^{*}-\mathbf{x}_{1, p}{ }^{*}\right)+\mathbf{x}_{2}{ }^{*}+(-1)^{k-1} \cdot \mathbf{x}_{3}{ }^{*}
\end{aligned}
$$

for $k \geq 1$. 
$\mathbf{x}(k)$ is made up of components with frequencies $\Omega, \theta$, and $p \cdot \omega$, where $p=0,1, \cdots, M-1$. This is different from the non-overflow case, where there are only the frequency components $\Omega$ and $\theta$. Because more sinusoidal signals are superimposed together, the visual appearance for the periodic symbolic sequence case may be different from that of the non-overflow case. For example, if overflow does not occur at a certain initial condition $\mathbf{x}(0)$, and gives periodic symbolic sequences for another initial condition $\mathbf{x}^{\prime}(0)$, while $\Omega$ and $\theta$ are unchanged, the visual appearance of periodic symbolic sequence case may be different from that of the non-overflow case. This change of the visual appearance of the trajectory pattern does not occur in the autonomous and step response cases because only elliptical pattern occurs in both the type I and type II trajectory for the autonomous and step response cases. Figure 5 shows some simulation examples of the sinusoidal response case when the symbolic sequences are periodic.

\section{B. Set of initial conditions}

Since

$$
\sum_{p=1}^{M-1} \mathbf{T}_{p \cdot \omega} \cdot \widehat{\mathbf{A}}_{p \cdot \omega}{ }^{k} \cdot \mathbf{T}_{p \cdot \omega}{ }^{-1} \cdot\left(\mathbf{x}_{2, p}{ }^{*}-\mathbf{x}_{1, p}{ }^{*}\right)
$$

$\sum_{p=1}^{M-1} \mathbf{T}_{p \cdot \omega} \cdot \widehat{\mathbf{A}}_{p \cdot \omega}{ }^{k} \cdot \mathbf{T}_{p \cdot \omega}{ }^{-1} \cdot\left(\mathbf{x}_{2, p}{ }^{*}-\mathbf{x}_{1, p}{ }^{*}\right)$ is periodic with period $M$, these terms are $p \neq \frac{M}{2}$

dependent on each other. Hence, just the state space technique is not sufficient to efficiently find out the set of initial conditions. Instead, the set of initial conditions can be found using a frequency-domain approach as well.

$$
\text { Let } \xi_{i}(n)=\left\{\begin{array}{cc}
\xi_{i,-n} & -(M-1) \leq n \leq-1 \\
0 & \text { otherwise }
\end{array} \text { for } i=0,1, \text { and } Z_{1}(\varpi) \text { and } Z_{2}(\varpi)\right. \text { be }
$$

the Fourier transforms of $\xi_{1}(n)$ and $\xi_{2}(n)$, respectively.

If $M$ is odd, then we have 


$$
\begin{aligned}
& \left\{\mathbf{x}(0):\left\|\mathbf{T}_{\Omega}{ }^{-1} \cdot\left(\mathbf{x}(0)+\mathbf{x}_{1}{ }^{*}-\mathbf{x}_{2}{ }^{*}+\sum_{p=1}^{M-1}\left(\mathbf{x}_{1, p}{ }^{*}-\mathbf{x}_{2, p}{ }^{*}\right)\right)\right\|<\right. \\
& 1-\left\|\mathbf{T}_{\theta}{ }^{-1} \cdot \mathbf{x}_{1}{ }^{*}\right\|-\max _{k \in\{0,1, \cdots, M-1\}} \mid \frac{Z_{1}(k \cdot \omega)+Z_{1}(-k \cdot \omega)}{2}+\frac{Z_{2}(k \cdot \omega)-Z_{2}(-k \cdot \omega)}{2 \cdot j}+\frac{b_{0}}{1-\cos \Omega} \|
\end{aligned}
$$

If $M$ is even, then we have

$$
\begin{aligned}
& \left\{\mathbf{x}(0): \| \mathbf{T}_{\Omega}{ }^{-1} \cdot\left(\mathbf{x}(0)+\mathbf{x}_{1}{ }^{*}-\mathbf{x}_{2}{ }^{*}+\mathbf{x}_{3}{ }^{*}+\sum_{\substack{p=1 \\
p \neq \frac{M}{2}}}^{M-1}\left(\mathbf{x}_{1, p}{ }^{*}-\mathbf{x}_{2, p}{ }^{*}\right) \|<\right.\right. \\
& 1-\left\|\mathbf{T}_{\theta}^{-1} \cdot \mathbf{x}_{1}{ }^{*}\right\|-\max _{k \in\{0,1, \cdots, M-1\}}\left|\frac{Z_{1}^{\prime}(k \cdot \omega)+Z_{1}^{\prime}(-k \cdot \omega)}{2}+\frac{Z_{2}^{\prime}(k \cdot \omega)-Z_{2}^{\prime}(-k \cdot \omega)}{2 \cdot j}+\frac{b_{0}}{1-\cos \Omega}-\frac{(-1)^{k-1} \cdot b_{\frac{M}{2}}}{1+\cos \Omega}\right|
\end{aligned}
$$

This corresponds to a set of elliptical regions. If $M$ is odd, then the centers are located at $-\mathbf{x}_{1}{ }^{*}+\mathbf{x}_{2}{ }^{*}-\sum_{p=1}^{M-1}\left(\mathbf{x}_{1, p}{ }^{*}-\mathbf{x}_{2, p}{ }^{*}\right)$ and the sizes of those ellipses depend on $1-\left\|\mathbf{T}_{\theta}^{-1} \cdot \mathbf{x}_{1}^{*}\right\|-\max _{k \in\{0,1, \cdots, M-1\}}\left|\frac{Z_{1}(k \cdot \omega)+Z_{1}(-k \cdot \omega)}{2}+\frac{Z_{2}(k \cdot \omega)-Z_{2}(-k \cdot \omega)}{2 \cdot j}+\frac{b_{0}}{1-\cos \Omega}\right| . \quad$ If $M$ is even, then the centers are located at $-\mathbf{x}_{1}{ }^{*}+\mathbf{x}_{2}{ }^{*}-\mathbf{x}_{3}{ }^{*}-\sum_{p=1}^{M-1}\left(\mathbf{x}_{1, p}{ }^{*}-\mathbf{x}_{2, p}{ }^{*}\right)$ and the $p \neq \frac{M}{2}$

sizes of those ellipses depend on $1-\left\|\mathbf{T}_{\theta}^{-1} \cdot \mathbf{x}_{1}^{*}\right\|-\max _{k \in\{0,1, \cdots, M-1\}}\left|\frac{Z_{1}^{\prime}(k \cdot \omega)+Z_{1}^{\prime}(-k \cdot \omega)}{2}+\frac{Z_{2}^{\prime}(k \cdot \omega)-Z_{2}^{\prime}(-k \cdot \omega)}{2 \cdot j}+\frac{b_{0}}{1-\cos \Omega}-\frac{(-1)^{k-1} \cdot b_{\frac{M}{2}}}{1+\cos \Omega}\right|$.

\section{Necessary conditions on filter parameters}

The necessary conditions for the periodic symbolic sequence case can be investigated by examining the conditions when the relation (9) or (10) is satisfied. To understand the expression (9) or (10) more, we can look at the expression term by term. For the first term, if those centers discussed in section IVB are inside the unit square $I^{2}[1]$, then one can select the initial condition $\mathbf{x}(0)$ as close to those centers as possible so that the above norms are close to zero. The second term is directly 
proportional to the amplitude of the input sinusoidal signal. As the amplitude of the input sinusoidal signal is small, then this norm is also small. However, the last term depends on $\quad \mathbf{T}_{p \cdot \omega}{ }^{-1} \cdot\left(\mathbf{x}_{2, p}{ }^{*}-\mathbf{x}_{1, p}{ }^{*}\right)=\frac{1}{\cos (p \cdot \omega)-\cos \Omega} \cdot\left[\begin{array}{l}b_{p} \cdot \cos (p \cdot \omega)-a_{p} \cdot \sin (p \cdot \omega) \\ b_{p} \cdot \sin (p \cdot \omega)+a_{p} \cdot \cos (p \cdot \omega)\end{array}\right]$, this implies that $\Omega \neq 2 \cdot \pi \cdot k \pm p \cdot \omega$, for $p \in\{1, \cdots, M-1\}$ and $M$ is odd, or $\Omega \neq 2 \cdot \pi \cdot k \pm p \cdot \omega$, for $p \in\{1, \cdots, M-1\} \backslash\left\{\frac{M}{2}\right\}$ and $M$ is even. In other words, the natural frequency of the digital filter does not equal to one of the harmonic frequencies of the symbolic sequences.

D. Simulation results for aperiodic symbolic sequence case

When the symbolic sequences are eventually periodic or aperiodic, the dynamic of the system is so complex that only some simulation results are obtained. For the autonomous and step response cases, it is reported in [1], [2] that there is an elliptical fractal pattern on the phase portrait if the symbolic sequences are aperiodic. However, we have conducted extensive simulations and find that both an elliptical fractal pattern and a rather random-like chaotic behavior may be exhibited for the sinusoidal response case. Figure 6 illustrates the simulation results.

\section{CONCLUSION}

The main focus of this paper is the analysis of second-order digital filters with two's complement arithmetic, when there are sinusoidal inputs. For the autonomous and step response cases, the trajectories can be classified by their visual appearances [1]. However, such classification is not applicable for the sinusoidal response case. Instead, we analyze the system behaviors by means of the periodicity of the symbolic 
sequences. Hence, instead of study the behaviors, the effect of the initial conditions and the filter parameters on different types of trajectories, we study those behaviors on different types of symbolic sequences.

Even when overflow does not occur, many trajectory patterns may be exhibited on the phase portrait. One interesting example is that there are several ellipses on the phase portrait, which appears to correspond to the type II trajectory for the autonomous and step response cases [1], [2]. The condition for the state vector to be periodic is investigated. The set of initial conditions and the set of filter and input parameters are also found.

When overflow occurs, the system dynamics is very complex. If the corresponding symbolic sequences are periodic, some analytical results are obtained by employing the frequency domain approach. Based on this approach, the set of initial conditions and the necessary conditions on the filter parameters are derived.

When the symbolic sequences are aperiodic, both an elliptical fractal pattern and a rather random-like chaotic behavior may be exhibited on the phase portrait instead of only the elliptical fractal type of pattern for the type III trajectories found in the autonomous and step response cases [1], [2]. Some simulation results are given.

\section{REFERENCES}

[1] L. O. Chua and T. Lin, "Chaos in digital filters," IEEE Transactions on Circuits and Systems, vol. 35, no. 6, pp. 648-658, June 1988.

[2] W. K. Ling, P. K. S. Tam and X. Yu, "Step response of a second-order digital filter with two's complement arithmetic," IEEE Transactions on Circuits and Systems - I: Fundamental Theory and Applications, vol. 50, no. 4, April 2003.

[3] R. L. Devaney, An introduction to chaotic dynamical systems, Addison-Wesley 
Publishing Company, Inc., 1986. 

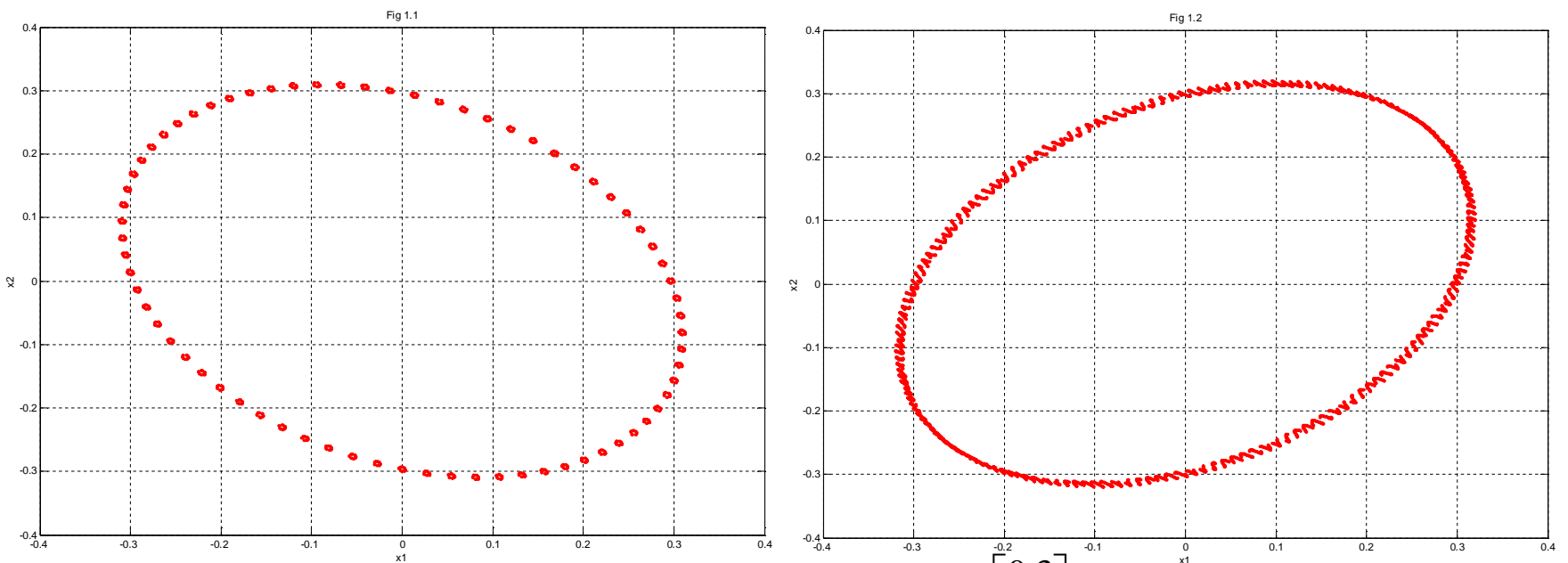

Fig. 1. Phase portrait of the system when $b=-1, \quad \mathbf{x}(0)=\left[\begin{array}{c}0.3 \\ 0\end{array}\right]^{0.1}$. (Fig 1.1$) \quad c=0.01$,

$\theta=-\pi+5$ and $\Omega=-\pi-1.3$. (Fig 1.2) $c=0.01, \theta=-\pi+6$ and $\Omega=-\pi-1.9$.
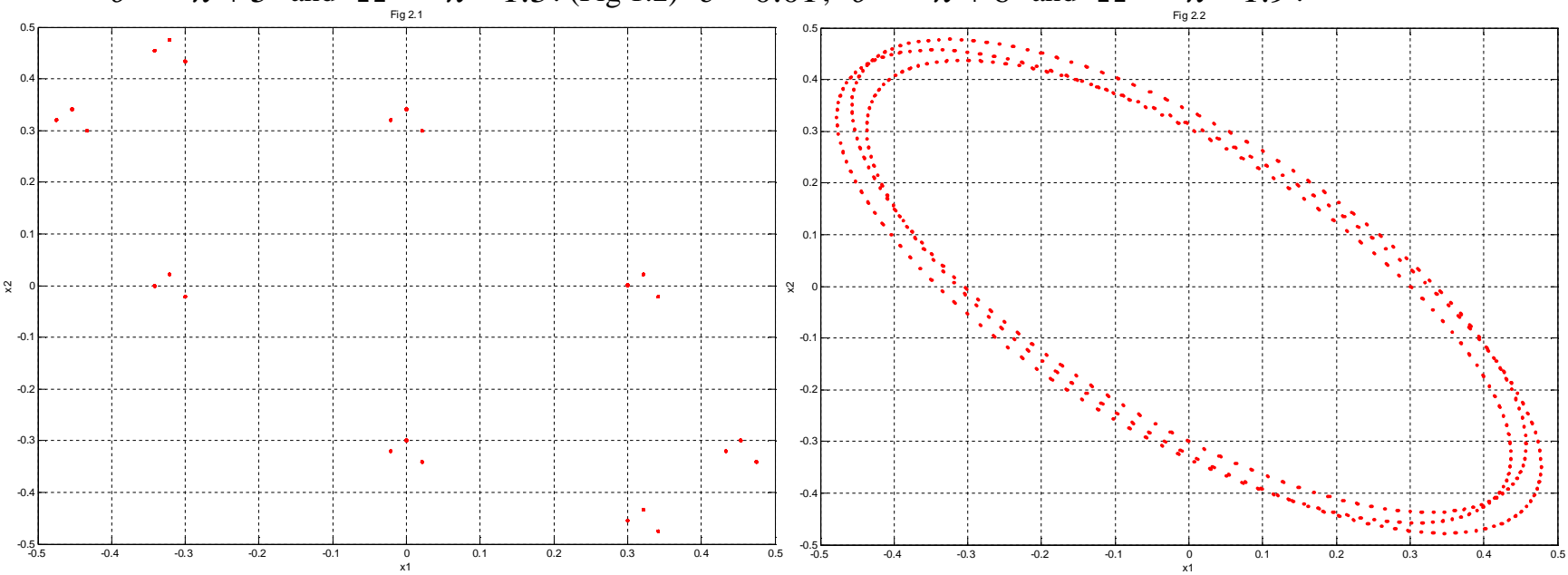

Fig. 2. Phase portrait of the system when $c=0.01, b=-1, \mathbf{x}(0)=\left[\begin{array}{c}0.3 \\ 0\end{array}\right], \theta=\frac{2 \cdot \pi}{3}$, and different values of $\Omega$. (Fig 2.1) $\Omega=\frac{3 \cdot \pi}{4}$. (Fig 2.2) $\Omega=\frac{3 \cdot \pi}{4}+0.01$.

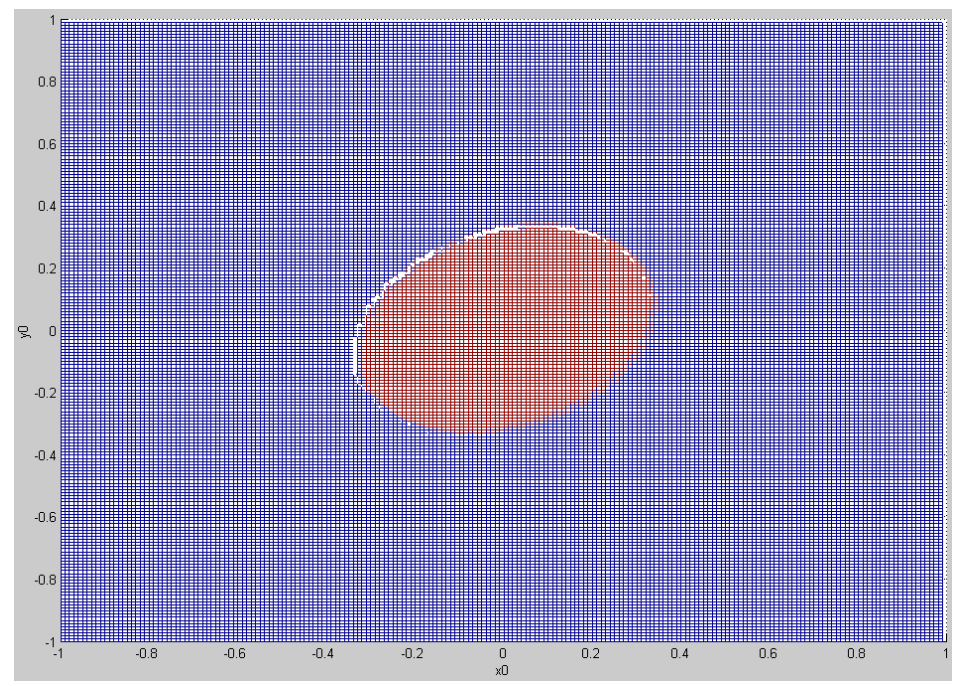

Fig. 3. Set of initial conditions for no overflow to occur, when $a=0.5, b=-1$, $c=1$ and $\theta=0.001 \cdot \pi$. 


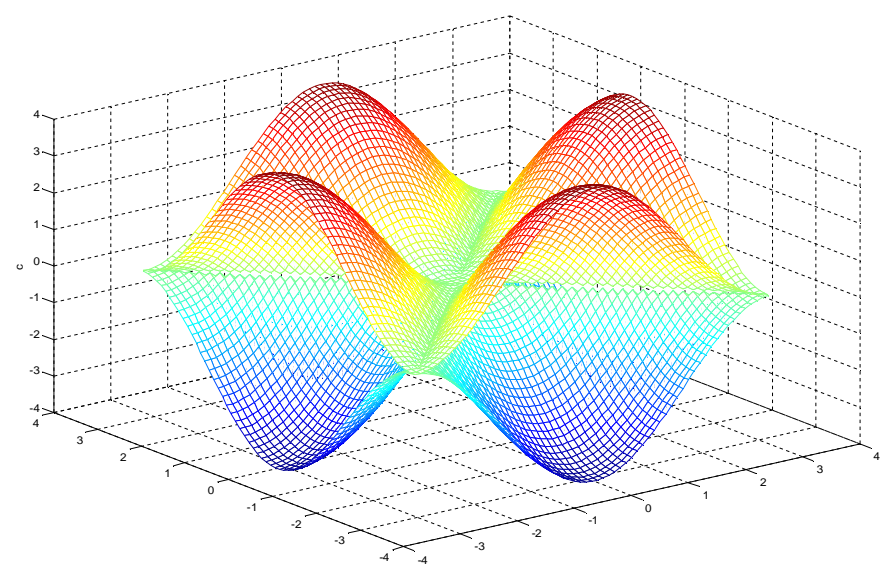

Fig. 4. Surface of the parameter region in which overflow may not occur when $b=-1$.
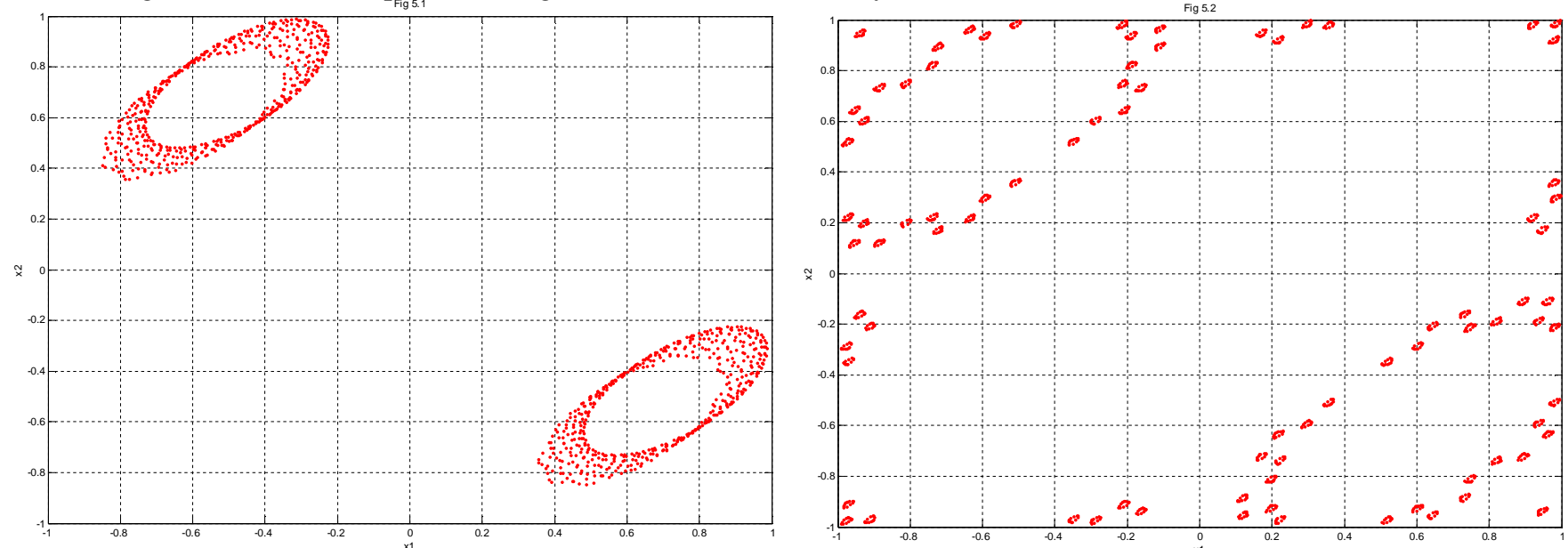

Fig. 5. Phase portrait for the system with different initial conditions, input and filter parameters. (Fig

5.1) $a=1.3, b=-1, \mathbf{x}(0)=\left[\begin{array}{c}0.5 \\ -0.5\end{array}\right], c=0.09, \theta=0.001 \cdot \pi$, the period is 2 . (Fig 5.2) $a=1.3, b=-1, \mathbf{x}(0)=\left[\begin{array}{l}-0.99 \\ -0.99\end{array}\right], c=0.005, \theta=0.001 \cdot \pi$, period is 84.
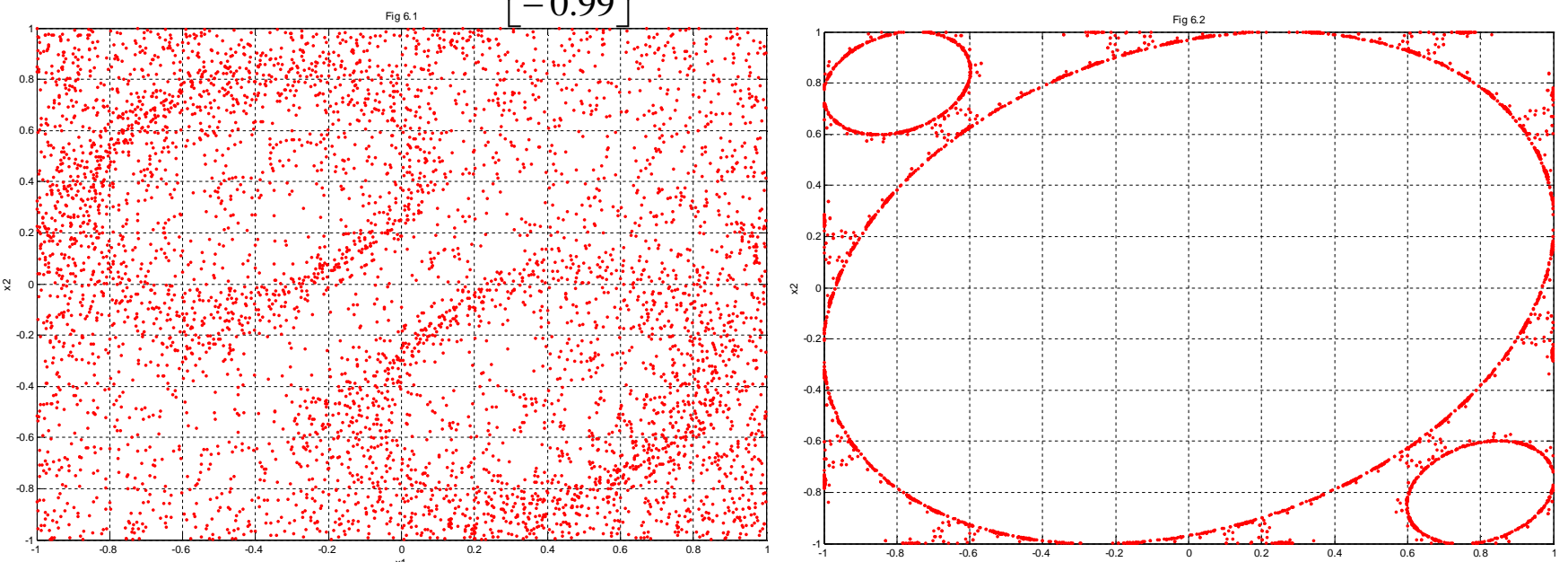

Fig. 6. Phase portrait for the system with different initial conditions, input and filter parameters. (fig 6.1)

$a=0.5, b=-1, \mathbf{x}(0)=\left[\begin{array}{c}-0.9999 \\ 0.9999\end{array}\right], c=1$ and $\theta=0.001 \cdot \pi$. (fig 6.2) $a=0.5, b=-1$,

$\mathbf{x}(0)=\left[\begin{array}{c}-0.6135 \\ 0.6135\end{array}\right], c=10^{-6}$ and $\theta=0.001 \cdot \pi$ 